\title{
A signature of ferroptosis-related genes in the prognosis and tumor microenvironment of Cervical squamous cell carcinoma and endocervical adenocarcinoma
}

\section{Dongmei Zhao}

Sichuan University West China Second University Hospital

Xiuli Qing ( $\square$ wintersweet2021@163.com )

West China second hospital https://orcid.org/0000-0003-0477-6474

\section{Research article}

Keywords: Cervical squamous cell carcinoma and endocervical adenocarcinoma, ferroptosis, prognosis, tumor microenvironment, TCGA

Posted Date: February 21st, 2022

DOI: https://doi.org/10.21203/rs.3.rs-934260/v1

License: (c) (i) This work is licensed under a Creative Commons Attribution 4.0 International License.

Read Full License 


\section{Abstract}

Background: The role of iron death in cervical squamous cell carcinoma and endocervical adenocarcinoma (CESC) is unclear. This study is based on the TCGA data set to explore the expression of ferroptosis-related genes (FRGs) in CESC and its clinical value.

Methods: We download the transcriptome and clinical data of CESC patients from the Cancer Genome Atlas of America (TCGA). R 4.4.0 software was used to analyze the differential expression of FRGs (DEFGs) and survival data to construct a risk prognostic model, determine the signature related to ferroptosis, and calculate the tumor microenvironment score of CESC. Then we analyzed the correlation between the ferroptosis-related signature, patient prognosis and tumor microenvironment score. GEPIA database and HPA database verified the differences in the expression and survival of DFRGs in normal cervical tissues and CESC tissues.

Results: Compared with normal cervical tissues, there were 14 DEFGs in CESC, 12 were up-regulated and 2 were down-regulated. Finally, four prognostic-related DEFGs (ACACA, PHKG2, SQLE and TFRC) were obtained to construct a prognostic risk model. It was found that the survival time was longer in the lowrisk group ( $\mathrm{HR}=0.444,95 \% \mathrm{Cl}$ : 0.279-0.707), and the stromal score, immune score and estimated score were higher $(\mathrm{P}<0.05)$. The verification in the GEPIA database and HPA database is basically consistent with our results.

Conclusion: The ferroptosis-related signature is significantly correlated with the survival and prognosis of CESC patients and the tumor microenvironment, suggesting that 4 DEFGs may be potential therapeutic targets and prognostic markers of CESC.

\section{Introduction}

Cervical cancer is the third most common cancer and the fourth leading cause of cancer-related mortality among women in the world. There are 530,000 cases and nearly 275,000 deaths worldwide each year, which is higher than other gynecological tumors. The mortality rate of cervical cancer ranks second in the mortality rate of female malignant tumors and the first in female reproductive organ tumors [1]. Among them, cervical squamous cell carcinoma and endocervical adenocarcinoma (CESC) account for more than $95 \%$ of cervical cancer [2]. In recent years, due to the progress of cervical cytology and cervical biopsy, better detection methods have been used to diagnose early and locally advanced cervical cancer. The main treatment methods for cervical cancer are surgery, radiotherapy and chemotherapy, and targeted therapy. Although cervical cancer screening and advanced medical methods have reduced the incidence of cervical cancer by $40-50 \%$ [3]. However, cervical cancer is still a major public health problem, and the prognosis of patients is poor, especially in advanced cases [4]. Tumor microenvironment plays an important role in tumor occurrence, metastasis and drug resistance. The main components of the tumor microenvironment of CESC are stromal components and immune components with low oxygen, low $\mathrm{pH}$, and high interstitial hydraulic pressure. There are also a large number of growth factors, 
chemokines and various proteolytic enzymes in the living environment of CESC tumor cells. This feature is very conducive to the proliferation, invasion, and invasion of CESC tumor cells. Adhesion, angiogenesis and drug resistance [5]. Therefore, looking for curative effect prediction, prognostic markers and the effected factors of tumor microenvironment is very important for patients with cervical cancer.

Ferroptosis is a cell death method first proposed in 2012, and it is related to the imbalance of redox homeostasis in the cell. Different from the traditional methods of death such as apoptosis and necrosis, the increase of intracellular free iron and lipid peroxidation are the unique metabolic characteristics of ferroptosis [6]. A large number of studies have shown that compared to normal cells, tumor cells require more iron to maintain growth, and this feature makes tumor cells more sensitive to ferroptosis [7].

Therefore, using ferroptosis to kill tumors directly and indirectly is a new treatment strategy. At the same time, biomarkers related to ferroptosis can also be used to predict tumor efficacy and prognosis [8]. Sorafenib can induce an increase in lipid oxidation levels for leading to cell death in liver cancer cells, Ferrostatin 1 as a specific inhibitor of ferroptosis, can prevent this effect, while inhibitors of apoptosis and necrosis cannot save this effect. Therefore, it can be considered that the way sorafenib induces the death of liver cancer cells is related to ferroptosis [9]. When ferroptosis inducers such as Erastin inhibit the Xcsystem activity of diffuse large B-cell lymphoma (DLBCL) cells, the cystine that synthesizes reduced glutathione cannot enter the cell due to the lack of its own transsulfuration pathway, resulting in peroxide of lipids cannot be reduced in time and effectively, thereby inducing the ferroptosis of DLBCL cells [10]. However, the role of ferroptosis-related genes (FRGs) in cervical cancer is not yet known.

Therefore, we used bioinformatics methods to comprehensively analyze the data of CESC downloaded from TCGA, and screened out the differentially expressed ferroptosis-related genes (DEFGs) related to prognosis, and constructed an prognostic risk model of CESC according to DEFGs and explored the role of these prognostic DEFGs in the tumor microenvironment of CESC. It aims to provide new treatment targets and prognostic markers for CESC patients.

\section{Materials And Methods}

\section{Data collection and analysis}

All CESC transcriptome and clinical data were downloaded from the TCGA database. A total of 308 tissue samples were obtained, including 305 tumor tissues and 3 normal tissues. $60 \mathrm{FRGs}$ were downloaded from Molecular Signatures Database v7.2 (https://www.gsea-msigdb.org/gsea/msigdb/index.jsp). These FRGs were screened out in CESC and the DEFGs were obtained with FDRfilter $=0.05$ and LogFCfilter $=0$ as the screening criteria. Unicox was used to screen out the prognostic-related DEFGs through the $R$ package "limma". The heat map was used to visualize the results through the R package "pheatmap". The correlation between the the prognostic-related DEFGs was analyzed by the R package "corrplot".

\section{Prognosis signature of ferroptosis-related genes (FRGs) in CESC}


We use Lasso regression to analyze the dimensionality reduction of the prognostic-related DEFGs and obtain the key prognostic-related DEFGs through the R package "glmnet" to form a ferroptosis-related signature to construct a prognostic risk model. The calculation formula is risk score $=$ gene $1^{\star}$ genecoef 1 + gene $2{ }^{*}$ genecoef $2+\ldots+$ gene $N^{*}$ genecoef $N$. Analyze the relationship between each prognostic-related DEFGs and risk model and survival time through the R package "survival". The ROC curve evaluated the sensitivity and specificity of the risk model by calculating the area under the curve through the $\mathrm{R}$ package "timeROC".

\section{Tumor microenvironment score related to prognosis signature of FRGs in CESC}

The tumor microenvironment score is composed of stromal score, immune score and estimate score and is calculated by the R package "estimate". And compare the survival differences between high and low groups, as well as the differences in tumor microenvironment scores in the risk model and the expression levels of the four key prognostic-related DEFGs.

\section{The verification of the key prognostic-related DEFGs in GEPIA database and HPA database}

The expression of key prognostic-related DEFGs and the relationship to survival in the CESC were verified in the GEPIA 2.0 database (http://gepia2.cancer-pku.cn/\#index) and the Human Protein Atlas (HPA) database (https://www.proteinatlas.org/).

\section{Statistical analysis}

All research results are subject to $P<0.05$ as statistically significant. The survival curve uses the nonparametric Kaplan-Meier method. $T$ test was used for analyzing DEFGs. The correlation analysis used univariate and multivariate cox.

\section{Results}

\section{The screening and analysis of DEFGs in CESC}

We got a total of 14 DEFGs, of which ACACA, FANCD2, GCLM, GSS, HMGCR, MT1G, PGD, PHKG2, SLC1A5, SQLE STEAP3 and TFRC are up-regulated, and CRYAB and ZEB1 are down-regulated. The 5 prognostic-related DEFGs (HMGCR, TFRC, PHKG2, ACACA, SQLE) obtained by unicox were all upregulated. (Fig. 1. A) PHKG2 was negatively correlated with the remaining 4 genes; HMGCR, TFRC, ACACA and SQLE were all positively correlated. (Fig. 1. B)

\section{Survival and prognosis signature of DEFGs in CESC}

The ACACA, HMGCR, SQLE, and TFRC were all risk factors for CESC (HR > 1). On the contrast, PHKG2 was the protective factor for CESC $(H R=0.003)$. (Fig. 2. A) Lasso regression constructed a risk signature 
related to ferroptosis and divided all CESC samples into high and low risk groups according to the median risk score $($ Risk score $=$ ACACA $1.699890776-$ PHKG2*2.59493828786383 +

SQLE*0.473474839748202 + TFRC*1.74887195824355). The overall survival in the low-risk group was significantly longer than that in the high-risk group ( $\mathrm{HR}=0.444,95 \% \mathrm{Cl}$ : $0.279-0.707)$. (Fig. 2. B) The ROC curve showed that the risk model predicts that the AUC for 1-year survival was 0.772 , the AUC for 2-year survival was 0.683 , and the AUC for 3-year survival was 0.650 (Fig. 2. C). It showed that the risk signature we constructed has good sensitivity. The risk signature was validated by GSE44001 set. The AUC for 1year survival was 0.667 , the AUC for 2-year survival was 0.715 , and the AUC for 3-year survival was 0.680 (Fig. 2. D). The survival curve showed that survival time of the patients in the low risk was longer (Fig. 2. E).

ACACA (Fig. 3. A), SQLE (Fig. 3. C), and TFRC (Fig. 3.D) with low expression in CESC survived longer. The survival time was longer in high expression of PHKG2. (Fig. 3. B)

\section{The correlation of prognosis signature and tumor microenvironment score in CESC}

The tumor microenvironment score was calculated and the CESC samples were divided into high and low score groups. We found no significant difference in survival in stromal score, immune score, and estimated score. (Fig. 4. A-C) In the low-risk group, the stromal score, immune score and estimated score were all higher than those in the high-risk group. (Fig. 4. D-F)

\section{The correlation of four key prognostic-related DEFGs and tumor microenvironment score in CESC}

We also studied the relationship between the tumor microenvironment score and the expression levels of 4 key prognostic-related DEFGs. In the low expression group of ACACA (Fig. 5. A) and SQLE (Fig. 5. D), the stromal score, immune score and estimated score were higher than those in the high risk group. There was no significant difference in the tumor microenvironment score between the high and low expression groups of PHKG2 (Fig. 5. B) and SQLE (Fig. 5. C).

The verification of four key prognostic-related DEFGs in the CESC from the GEPIA database and HPA database

The expression level of four key prognostic-related DEFGs was higher in the CESC group compared to the normal cervical tissue group. (Fig. 6. A-D) The overall survival of ACACA, SQLE and TFRC was longer in the low expression group (Fig. 6. E, G-H), and the overall survival of PHKG2 was longer in the high expression group of CSES. (Fig. 6. F) Additionally, the immunohistochemical analysis of ACACA, SQLE and TFRC was from HPA database except PHKG2 due to lacking of its data. (Fig. 7) The verification of these results was consistent with our results. 


\section{Discussion}

The incidence of CESC patients tends to be younger. Although CESC screening and diagnosis methods have been gradually improved, and surgical treatment and radiotherapy and chemotherapy have made great progress, the long-term survival rate and quality of life of patients still need to be improved [11]. Starting from the genetic level to study the mechanism of the occurrence and development of CESC is conducive to the discovery of new molecular targets, and provides research basis for the precise treatment and prognosis of CESC [12]. Our research showed for the first time that DEFGs and prognostic risk signature based on DEFGs were good predictors of CESC. Moreover, DEFGs were also potential therapeutic targets of CESC, and at the same time affected the stromal components and immune components of the tumor microenvironment in CESC, indicating that DEFGs may be closely related to the occurrence, development, invasion and metastasis of CESC. At the same time, DEFGs may also be the biomarkers for predicting the efficacy of immunotherapy.

A large number of studies have shown that ferroptosis can kill tumor cells and inhibit tumor growth. On the contrary, tumor cells inhibit ferroptosis through self-protection mechanisms to reduce cell death [13]. The main feature of ferroptosis is the accumulation of lipid-ROS (L-ROS) and fatal ROS from iron metabolism, which can be inhibited by iron chelators and lipid peroxidation inhibitors [14]. Gluathione peroxidase 4, (GPX4), heat shock protein $\beta 1$ (HSBP1) and nuclear factor-f2 (Nrf2) play a negative regulatory role on ferroptosis by limiting the production of ROS and reducing the uptake of iron by cells [15]. NADPH oxidase and P53 play a positive regulatory role by promoting the production of ROS. The current research on the related mechanism of ferroptosis focuses on the direction of oxidative damage, the direction of iron metabolism regulation, and the relevant targets discovered in experiments [16]. Studies have shown that the sensitivity of cells to ferroptosis can be changed by adjusting the content of intracellular iron. By increasing transferrin and TFRI, intracellular iron can be increased and the occurrence of ferroptosis can be promoted. Among them, heat shock protein has become a relevant target for initial results [17]. However, research on the relationship between ferroptosis and tumors is still mainly limited to cell and animal experiments.

In our research, ACACA was reported for the first time in relation to ferroptosis in the role of CESC. PHKG2, SQLE and TRFC are also the first time that the potential role of ferroptosis-related pathways in the prognosis of CESC and the tumor microenvironment has been discovered. The full name of ACACA is acetyl-CoA carboxylase alpha. It is found to be highly expressed in prostate cancer. After ACACA is inhibited in in vivo experiments, the mitochondrial ATP production decreases significantly, and the ratio of $\mathrm{NAD+} / \mathrm{NADH}$ to ROS levels increases. Therefore, targeting ACACA genes and mitochondria to induce ferroptosis may be a new therapy for prostate cancer [18]. In invasive non-functional pituitary adenoma (NFPA), it was found that ACACA is one of the key regulators of phosphorylation-mediated changes in the signaling pathway network, and is a potential predictor/prognostic marker for patients with NFPA, as well as a potential therapeutic target [19]. 
Alectinib is an ALK inhibitor, which can be used to treat ALK-positive lung cancer, but it also targets PHKG2 (phosphorylase kinase gamma submit 2) for anti-tumor effects [20]. In patients with metastatic colorectal cancer (mCRC) treated with cetuximab and irinotecan (Cl), PHKG2 was significantly overexpressed, which can effectively predict the efficacy of $\mathrm{Cl}$ for $\mathrm{mCRC}$ [21]. PHKG2 has been verified to be differentially expressed in endometrial cancer tissues and is a potential marker for the diagnosis and identification of endometrial cancer [22].

The stable expression of SQLE leads to increased cholesterol synthesis and activates PI3K/Akt signaling, thereby regulating the stemness of breast cancer stem cells, which may overcome breast cancer chemotherapy resistance [23]. Metformin can inhibit the stemness of mCRC by down-regulating SQLE and affecting lipid metabolism through the mevalonate pathway [24]. SQLE is also a cholesterol metabolism molecule and a promising therapeutic target for tumors [25]. Current research mainly shows that SQLE is a new type of lipid metabolism regulator, which will affect tumor development and metastasis. Abnormal lipid metabolism is the core mechanism of ferroptosis, so it may also play an important role in tumor development and metastasis.

YTHDF1 enhances the expression of TFRC in head and neck squamous cell carcinomas (HPSCC) through an m6A-dependent mechanism, so TFRC is a key target gene for YTHDF1-mediated increase in iron metabolism. In the future, it is possible to target YTHDF1 and TFRC-mediated iron metabolism may be a promising treatment strategy for HPSCC [26]. We found that the high expression of ACACA, SQLE and TFRC in CESC was associated with a poor prognosis, while the high expression of PHKG2 was associated with a good prognosis. Therefore, ACACA, SQLE and TFRC can be inhibited, and the high expression of PHKG2 can effectively induce ferroptosis as a target for CSEC treatment and overcome chemotherapy resistance. At the same time, detecting their expression level and the ferroptosis-related signature constructed based on these DEFGs can predict the prognosis of CSEC.

Immune cells activated during immunotherapy can enhance the level of ferroptosis-specific lipid peroxidation in tumor cells, and the enhancement of ferroptosis contributes to the anti-tumor efficacy of immunotherapy [27]. Through further research, it is found that the mechanism of interferon gamma (IFN gamma) released by CD $8+T$ cells down-regulates the expression of System Xc-, impairing the absorption of cystine by tumor cells, thereby promoting lipid peroxidation and ferroptosis in tumor cells. Through the analysis of clinical tumor samples, the expression of System Xc- in cancer patients is negatively correlated with the number of CD8 + T cells, the expression of IFNY, and the prognosis of patients [28]. Both immunotherapy and radiotherapy require immune cells. Immune cells can change the sensitivity of tumor cells to radiotherapy by promoting ferroptosis. Immune cell-derived IFNY and radiation synergistically inhibit the expression of tumor SLC7A11 and induce tumor cell apoptosis, while radiotherapy activate ATM to inhibit the expression of SLC7A11, limit the uptake of tumor cystine, reduce glutathione, and increase lipid oxidative damage to mediate the iron vision of tumor cells. Biochemical and genetic suppression of ataxia telangiectasia mutant gene (ATM) can prevent the loss of SLC7A11 expression, reduce lipid oxidation, and rescue tumor cell ferroptosis caused by radiotherapy [29]. This study shows that ferroptosis is a previously unknown mechanism of tumor cell death after in vivo and in 
vitro radiotherapy, and that radiotherapy can make tumor cells sensitive to ferroptosis agonists both in vitro and in vivo, highlighting a new strategy for radiosensitizing tumors. Radiotherapy is the main treatment of CESC, so increasing the activity of ferroptosis during radiotherapy of CESC will be an effective way to improve the sensitivity of radiotherapy.

Inducing the secretion of key factors such as ROS produced by ferroptosis into the tumor microenvironment to enhance the anti-tumor effect is an effective step for tumor treatment or improving the efficacy [30]. We found that the low-risk group of ferroptosis-related signatures has a high TME score, and the TME score is also high when the expression of ACACA and SQLE is low, indicating that increasing the level of ferroptosis is indeed more conducive to enhancing the anti-tumor effect of CESC. At present, there are few studies on the relationship between the four key prognostic-related DEFGs and tumors. Therefore, the four key prognostic-related DEFGs are potential therapeutic targets of CESC and are also biomarkers for predicting efficacy and prognosis.

The limitations of this study are: (1) The sample is limited; (2) It lacks of the experiment verification in vivo or vitro. Therefore, we will further verify the results in the next research.

\section{Conclusion}

The ferroptosis-related signature is significantly correlated with the survival and prognosis of CESC patients and the tumor microenvironment, suggesting that 4 DEFGs may be potential therapeutic targets and prognostic markers of CESC.

\section{Declarations}

\section{ACKNOWLEDGMENTS}

Thank all scholars for developing public databases and contributing data, we could freely use the data for research. CONFLICT OF INTEREST

The authors declare that they have no competing interests.

\section{AUTHORS' CONTRIBUTIONS}

Dongmei Zhao drafted the manuscript. Xiuli Qing revised of the manuscript.

\section{ETHICS APPROVAL AND CONSENT TO PARTICIPATE}

The data in this study downloaded from public databases, did not involve human or animal organizations, and had no ethical issues.

\section{References}


1. Biyuan Xing, Jianfeng Guo, Yuhan Sheng, Gang Wu, Yingchao Zhao (2021) Human PapillomavirusNegative Cervical Cancer: A Comprehensive Review. Front Oncol. 10, 606335. doi:

10.3389/fonc. 2020.606335

2. Maria Isaguliants, Ekaterina Bayurova, Darya Avdoshina, Alla Kondrashova, Francesca Chiodi, Joel M Palefsky (2021) Oncogenic Effects of HIV-1 Proteins, Mechanisms Behind. Cancers (Basel). 13(2): 305. doi: $10.3390 /$ cancers 13020305

3. Zhen Dong, Renjian Hu, Yan Du, Li Tan, Lin Li, Juan Du, Longchang Bai, Yingkang Ma, Hongjuan Cui(2021) Immunodiagnosis and Immunotherapeutics Based on Human Papillomavirus for HPVInduced Cancers. Front Immunol. 11, 586796. doi: 10.3389/fimmu.2020.586796

4. Anthony H Taylor, Daniel Tortolani, Thangesweran Ayakannu, Justin C Konje, Mauro Maccarrone (2020) (Endo)Cannabinoids and Gynaecological Cancers. Cancers (Basel). 13(1): 37. doi: $10.3390 /$ cancers 13010037

5. Yuan H, Pratte J, Giardina C (2021) Ferroptosis and its potential as a therapeutic target. Biochem Pharmacol. 186, 114486. doi: 10.1016/j.bcp.2021.114486

6. Huizhen Zheng, Jun Jiang, Shujuan Xu, Wei Liu, Qianqian Xie, Xiaoming Cai, Jie Zhang, Sijin Liu, Ruibin Li (2021) Nanoparticle-induced ferroptosis: detection methods, mechanisms and applications. Nanoscale. 13(4): 2266-2285. doi: 10.1039/d0nr08478f

7. Hong-Fa Yan, Ting Zou, Qing-Zhang Tuo, Shuo Xu, Hua Li, Abdel Ali Belaidi, Peng Lei(2021) Ferroptosis: mechanisms and links with diseases. Signal Transduct Target Ther. 6(1): 49. doi: 10.1038/s41392-020-00428-9

8. Dionísio PA, Amaral JD, Rodrigues CMP (2021) Oxidative stress and regulated cell death in Parkinson's disease. Ageing Res Rev. 67, 101263. doi: 10.1016/j.arr.2021.101263

9. Xiaofang Sun, Zhanhui Ou, Ruochan Chen, Xiaohua Niu, De Chen, Rui Kang, Daolin Tang (2016) Activation of the p62-Keap1-NRF2 pathway protects against ferroptosis in hepatocellular carcinoma cells. Hepatology. 63(1): 173-184. doi: 10.1002/hep.28251

10. Yan Zhang, Hui Tan, Jacob D Daniels, Fereshteh Zandkarimi, Hengrui Liu, Lewis M Brown, Koji Uchida, Owen A O'Connor, Brent R Stockwell (2019) Imidazole Ketone Erastin Induces Ferroptosis and Slows Tumor Growth in a Mouse Lymphoma Model. Cell Chem Biol. 26(5): 623-633.e9. doi: 10.1016/j.chembiol.2019.01.008

11. Carse S, Bergant M, Schäfer G (2021) Advances in Targeting HPV Infection as Potential Alternative Prophylactic Means. Int J Mol Sci. 22(4): 2201. doi: 10.3390/ijms22042201

12. Natália Lourenço de Freitas, Maria Gabriela Deberaldini, Diana Gomes, Aline Renata Pavan, Ângela Sousa, Jean Leandro Dos Santos, Christiane P Soares (2021) Histone Deacetylase Inhibitors as Therapeutic Interventions on Cervical Cancer Induced by Human Papillomavirus. Front Cell Dev Biol. 8, 592868. doi: 10.3389/fcell.2020.592868

13. Yilei Zhang, Robert V Swanda, Litong Nie, Xiaoguang Liu, Chao Wang, Hyemin Lee, Guang Lei, Chao Mao, Pranavi Koppula, Weijie Cheng, Jie Zhang, Zhenna Xiao, Li Zhuang, Bingliang Fang, Junjie 
Chen, Shu-Bing Qian, Boyi Gan (2021) mTORC1 couples cyst(e)ine availability with GPX4 protein synthesis and ferroptosis regulation. Nat Commun. 12(1): 1589. doi: 10.1038/s41467-021-21841-w

14. Liying Zhao, Yanmei Peng, Sixiao He, Ru Li, Ziqing Wang, Junhao Huang, Xuetao Lei, Guoxin $\mathrm{Li}$, Qiang Ma (2021) Apatinib induced ferroptosis by lipid peroxidation in gastric cancer. Gastric Cancer. doi: 10.1007/s10120-021-01159-8

15. Michael A Badgley, Daniel M Kremer, H Carlo Maurer, Kathleen E DelGiorno, Ho-Joon Lee, Vinee Purohit, Irina R Sagalovskiy, Alice Ma, Jonathan Kapilian, Christina E M Firl, Amanda R Decker, Steve A Sastra, Carmine F Palermo, Leonardo R Andrade, Peter Sajjakulnukit, Li Zhang, Zachary P Tolstyka, Tal Hirschhorn, Candice Lamb, Tong Liu, Wei Gu, E Scott Seeley, Everett Stone, George Georgiou, Uri Manor, Alina luga, Geoffrey M Wahl, Brent R Stockwell, Costas A Lyssiotis, Kenneth P Olive (2020) Cysteine depletion induces pancreatic tumor ferroptosis in mice. Science. 368(6486): 85-89. doi: 10.1126/science.aaw9872

16. Jinghan Zhu, Yixiao Xiong, Yuxin Zhang, Jingyuan Wen, Ning Cai, Kun Cheng, Huifang Liang, Wanguang Zhang(2020) The Molecular Mechanisms of Regulating Oxidative Stress-Induced Ferroptosis and Therapeutic Strategy in Tumors. Oxid Med Cell Longev. 2020, 8810785. doi: $10.1155 / 2020 / 8810785$

17. Quanzhi Zhang, Hongmei Wu, Mingyang Zou, Ling Li, Qi Li, Caihong Sun, Wei Xia, Yonggang Cao, Lijie Wu (2019) Folic acid improves abnormal behavior via mitigation of oxidative stress, inflammation, and ferroptosis in the BTBR T+tf/J mouse model of autism. J Nutr Biochem. 71, 98109. doi: 10.1016/j.jnutbio.2019.05.002

18. Hui Zhang, Shaoyou Liu, Zhouda Cai, Weimin Dong, Jianheng Ye, Zhiduan Cai, Zhaodong Han, Yuxiang Liang, Yangjia Zhuo, Yong Luo, Xuejin Zhu, Yulin Deng, Yixun Zhang, Ren Liu, Yuanfa Feng, Jiarun Lai, Rui Zhou, Huijing Tan, Weide Zhong (2021) Down-regulation of ACACA suppresses the malignant progression of Prostate Cancer through inhibiting mitochondrial potential. J Cancer. 12(1): 232-243. doi: $10.7150 /$ jca. 49560

19. Dan Liu, Jiajia Li, Na Li, Miaolong Lu, Siqi Wen, Xianquan Zhan (2020) Integration of quantitative phosphoproteomics and transcriptomics revealed phosphorylation-mediated molecular events as useful tools for a potential patient stratification and personalized treatment of human nonfunctional pituitary adenomas. EPMA J. 11(3): 419-467. doi: 10.1007/s13167-020-00215-0

20. Yumura M, Nagano T, Nishimura Y (2020) Novel Multitarget Therapies for Lung Cancer and Respiratory Disease. Molecules. 25(17): 3987. doi: 10.3390/molecules25173987

21. Seung Tae Kim, Tae Jin Ahn, Eunjin Lee, In-Gu Do, Su Jin Lee, Se Hoon Park, Joon Oh Park, Young Suk Park, Ho Yeong Lim, Won Ki Kang, Suk Hyeong Kim, Jeeyun Lee, Hee Cheol Kim(2015) Exploratory biomarker analysis for treatment response in KRAS wild type metastatic colorectal cancer patients who received cetuximab plus irinotecan. BMC Cancer. 15, 747. doi: 10.1186/s12885015-1759-y

22. Eva Colas, Cristina Perez, Silvia Cabrera, Nuria Pedrola, Marta Monge, Josep Castellvi, Fernando Eyzaguirre, Jesus Gregorio, Anna Ruiz, Marta Llaurado, Marina Rigau, Marta Garcia, Tugçe 
Ertekin, Melania Montes, Rafael Lopez-Lopez, Ramon Carreras, Jordi Xercavins, Alicia Ortega, Tamara Maes, Elisabet Rosell, Andreas Doll, Miguel Abal, Jaume Reventos, Antonio GilMoreno (2011) Molecular markers of endometrial carcinoma detected in uterine aspirates. Int J Cancer. 129(10): 2435-44. doi: 10.1002/ijc.25901

23. Yilu Qin, Yixuan Hou, Shuiqing Liu, Pengpeng Zhu, Xueying Wan, Maojia Zhao, Meixi Peng, Huan Zeng, Qiao Li, Ting Jin, Xiaojiang Cui, Manran Liu (2020) A Novel Long Non-Coding RNA Inc030 Maintains Breast Cancer Stem Cell Stemness by Stabilizing SQLE mRNA and Increasing Cholesterol Synthesis. Adv Sci (Weinh). 8(2): 2002232. doi: 10.1002/advs.202002232

24. Yoojeong Seo, Janghyun Kim, Soo Jung Park, Jae Jun Park, Jae Hee Cheon, Won Ho Kim, Tae II Kim (2020) Metformin Suppresses Cancer Stem Cells through AMPK Activation and Inhibition of Protein Prenylation of the Mevalonate Pathway in Colorectal Cancer. Cancers (Basel). 12(9): 2554. doi: 10.3390/cancers1209255

25. Huanji Xu, Sheng Zhou, Qiulin Tang, Hongwei Xia, Feng Bi (2020) Cholesterol metabolism: New functions and therapeutic approaches in cancer. Biochim Biophys Acta Rev Cancer. 1874(1): 188394. doi: 10.1016/j.bbcan.2020.188394

26. Jing Ye, Zhanggui Wang, Xiaozhen Chen, Xiaohua Jiang, Zhihuai Dong, Sunhong Hu, Wenya Li, Yuehui Liu, Bing Liao, Weidong Han, Jiaying Shen, Mang Xiao (2020) YTHDF1-enhanced iron metabolism depends on TFRC m6A methylation. Theranostics. 10(26): 12072-12089. doi: $10.7150 /$ thno.51231

27. Alessandro Sacco, Anna Martina Battaglia, Cirino Botta, llenia Aversa, Serafina Mancuso, Francesco Costanzo, Flavia Biamonte (2021) Iron Metabolism in the Tumor Microenvironment-Implications for Anti-Cancer Immune Response. Cells. 10(2): 303. doi: 10.3390/cells10020303

28. Weimin Wang, Michael Green, Jae Eun Choi, Miguel Gijón, Paul D Kennedy, Jeffrey K Johnson, Peng Liao, Xueting Lang, Ilona Kryczek, Amanda Sell, Houjun Xia, Jiajia Zhou, Gaopeng Li, Jing Li, Wei Li, Shuang Wei, Linda Vatan, Hongjuan Zhang, Wojciech Szeliga, Wei Gu, Rebecca Liu, Theodore S Lawrence, Candice Lamb, Yuri Tanno, Marcin Cieslik, Everett Stone, George Georgiou, Timothy A Chan, Arul Chinnaiyan, Weiping Zou (2019) CD8+ T cells regulate tumour ferroptosis during cancer immunotherapy. Nature. 569(7755): 270-274. doi: 10.1038/s41586-019-1170-y.

29. Xueting Lang, Michael D Green, Weimin Wang, Jiali Yu, Jae Eun Choi, Long Jiang, Peng Liao, Jiajia Zhou, Qiang Zhang, Ania Dow, Anjali L Saripalli, Ilona Kryczek, Shuang Wei, Wojciech Szeliga, Linda Vatan, Everett M Stone, George Georgiou, Marcin Cieslik, Daniel R Wahl, Meredith A Morgan, Arul M Chinnaiyan, Theodore S Lawrence, Weiping Zou (2019) Radiotherapy and Immunotherapy Promote Tumoral Lipid Oxidation and Ferroptosis via Synergistic Repression of SLC7A11. Cancer Discov. 9(12): 1673-1685. doi: 10.1158/2159-8290.CD-19-0338.

30. Lesang Shen, Yunxiang Zhou, Haifei He, Wuzhen Chen, Cameron Lenahan, Xiaoyi Li, Yongchuan Deng, Anwen Shao, Jian Huang (2021) Crosstalk between Macrophages, T Cells, and Iron Metabolism in Tumor Microenvironment. Oxid Med Cell Longev. 2021, 8865791. doi: $10.1155 / 2021 / 8865791$ 
Figures
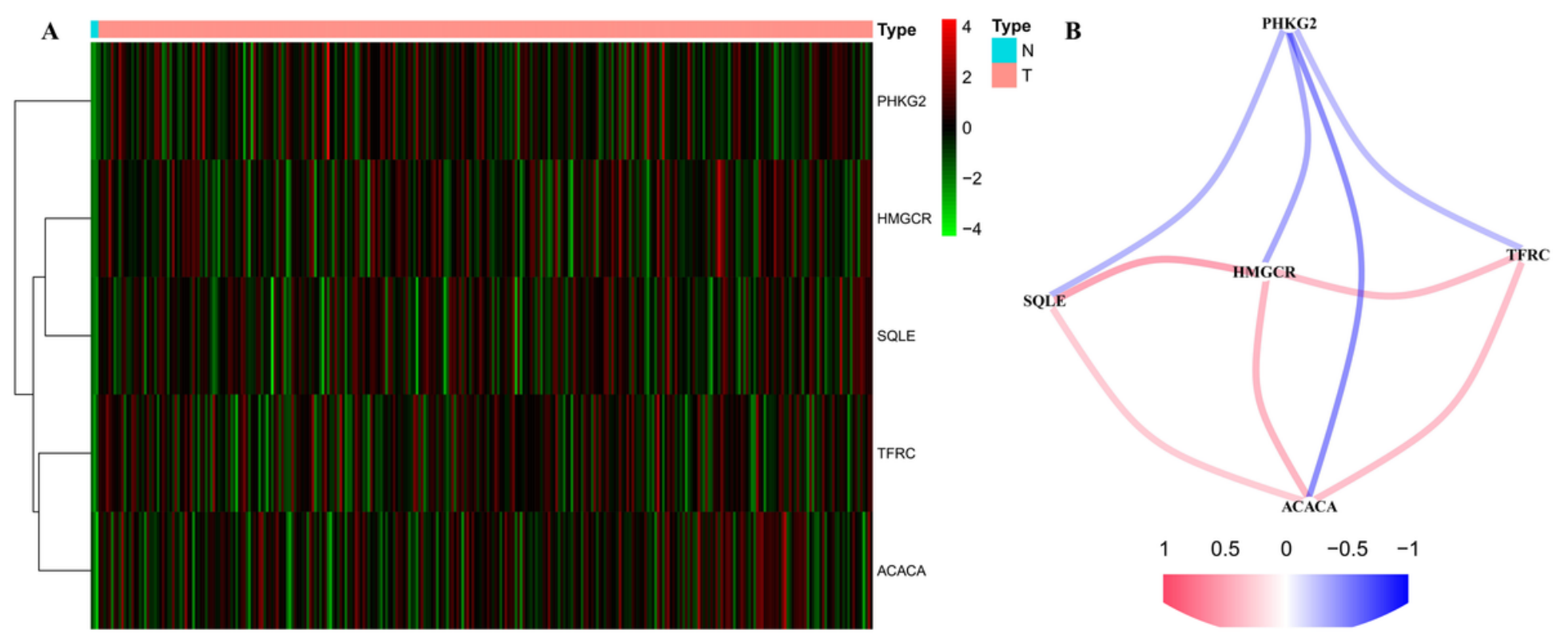

Figure 1

The expression of prognostic-related DEFGs in CESC with unicox analysis. A. The heatmap of 5 prognostic-related DEFGs in CESC. B. The correlation of 5 prognostic-related DEFGs in CESC. 
A

$\begin{array}{lrr} & \text { pvalue } & \text { Hazard ratio } \\ & & \\ \text { HMGCR } & 0.030 & 19.813(1.329-295.481) \\ \text { TFRC } & 0.001 & 26.277(3.649-189.247) \\ \text { PHKG2 } & 0.003 & 0.003(0.000-0.135) \\ & & \\ \text { ACACA } & 0.003 & 151.408(5.836-3928.269) \\ & & \\ \text { SQLE } & 0.026 & 7.486(1.270-44.113)\end{array}$

B

TCGA

Risk + High risk + Low risk

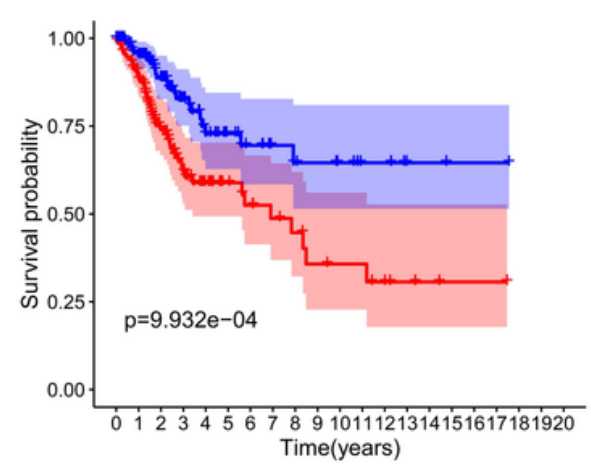

C

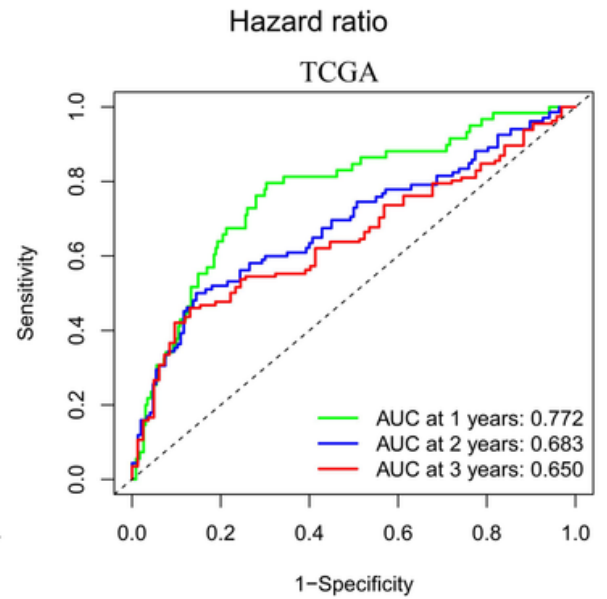

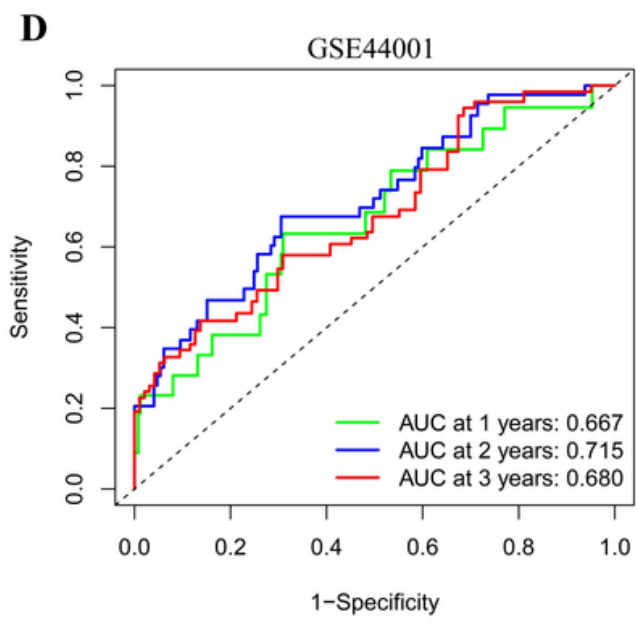

$\mathbf{E}$

GSE44001

Risk - High risk + Low risk

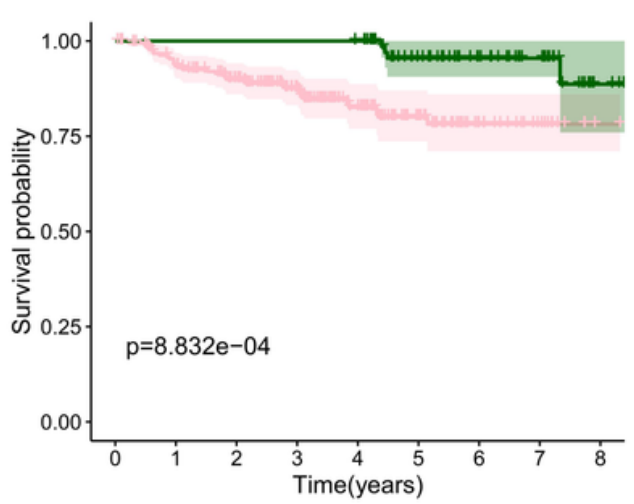

\section{Figure 2}

The prognosis signature of DEFGs in CESC. A. The forest plot of 5 prognostic-related DEFGs in CESC with unicox analysis. B. The survival curve of prognostic signature based on 4 key prognostic-related DEFGs with Lasso regression from TCGA set. C. The ROC curve of prognostic signature for predicting sensitivity and specificity in CESC from TCGA set. D. The ROC curve from GES44001 set. E. The survival curve from GES44001 set. 

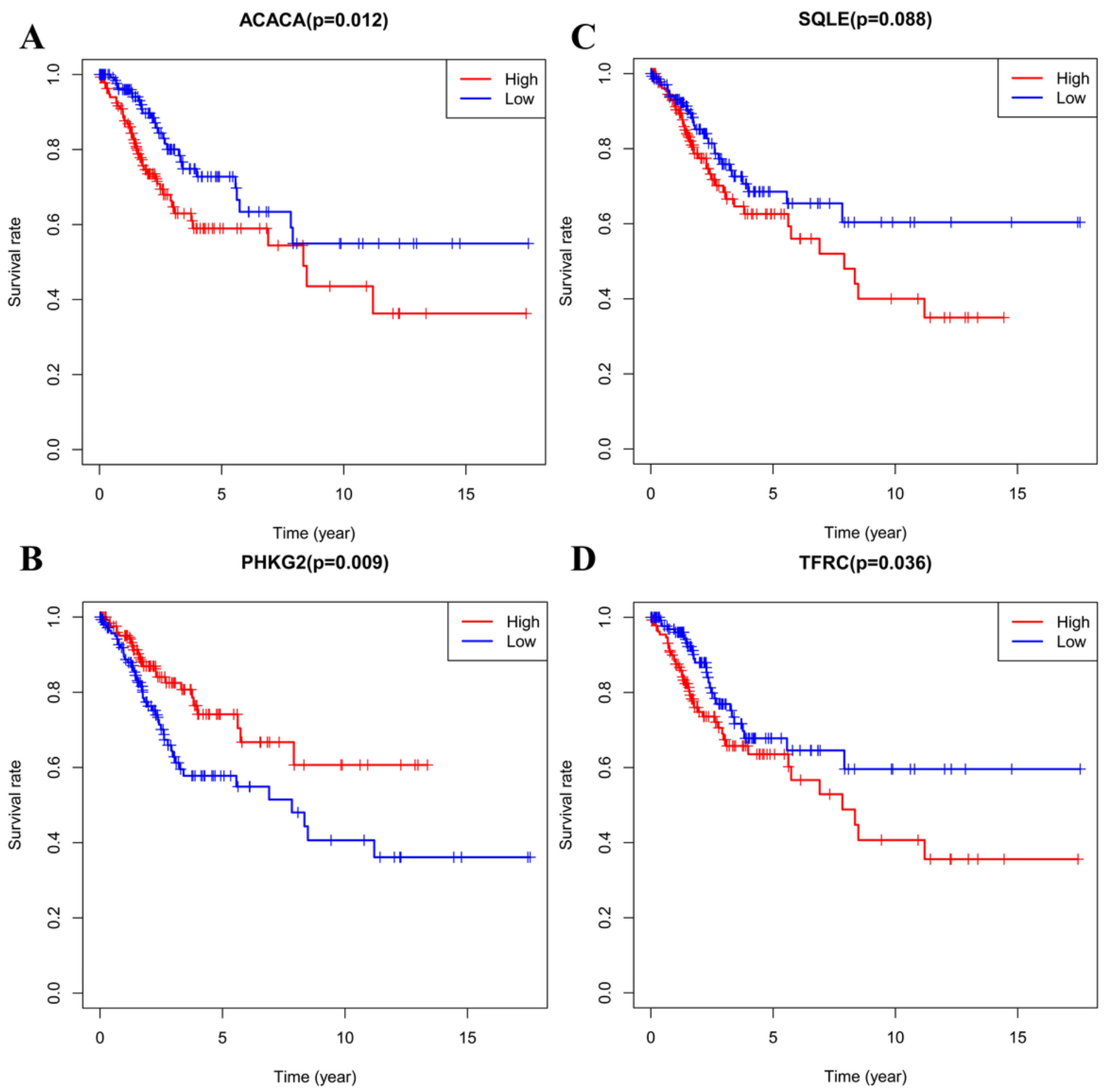

Figure 3

The survival curve of $\mathbf{4}$ key prognostic-related DEFGs in CESC. A. The survival curve of ACACA expression.

B. The survival curve of PHKG2 expression. C. The survival curve of SQLE expression. D. The survival curve of TFRC expression. 

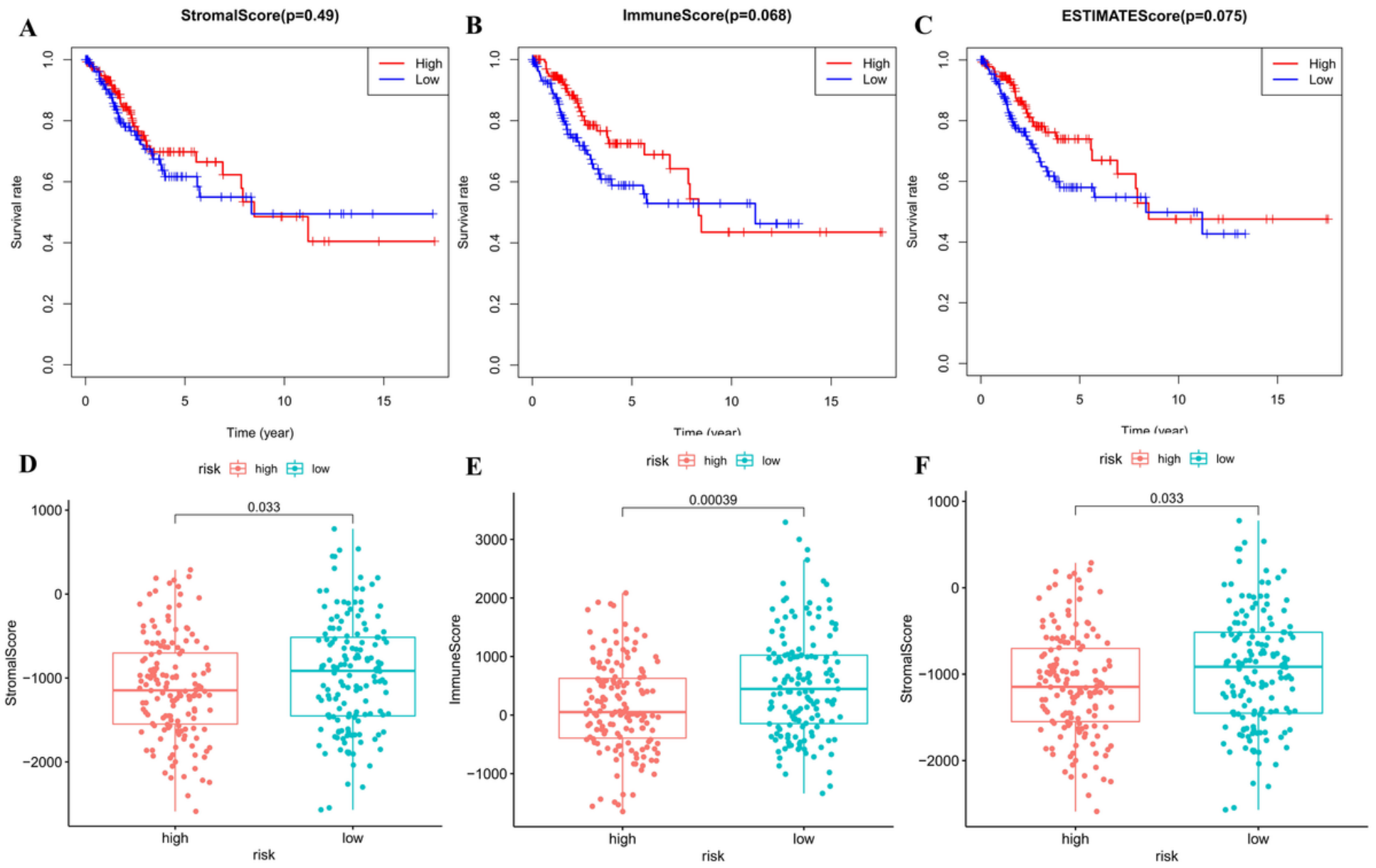

Figure 4

The correlation between tumor microenvironment score and prognosis signature of DEFGs in CESC. A. The survival curve of stromal score. B. The survival curve of immune score. C. The survival curve of estimate score. D. The correlation between stromal score and prognosis signature of DEFGs. E. The correlation between immune score and prognosis signature of DEFGs. F. The correlation between estimate score and prognosis signature of DEFGs. 

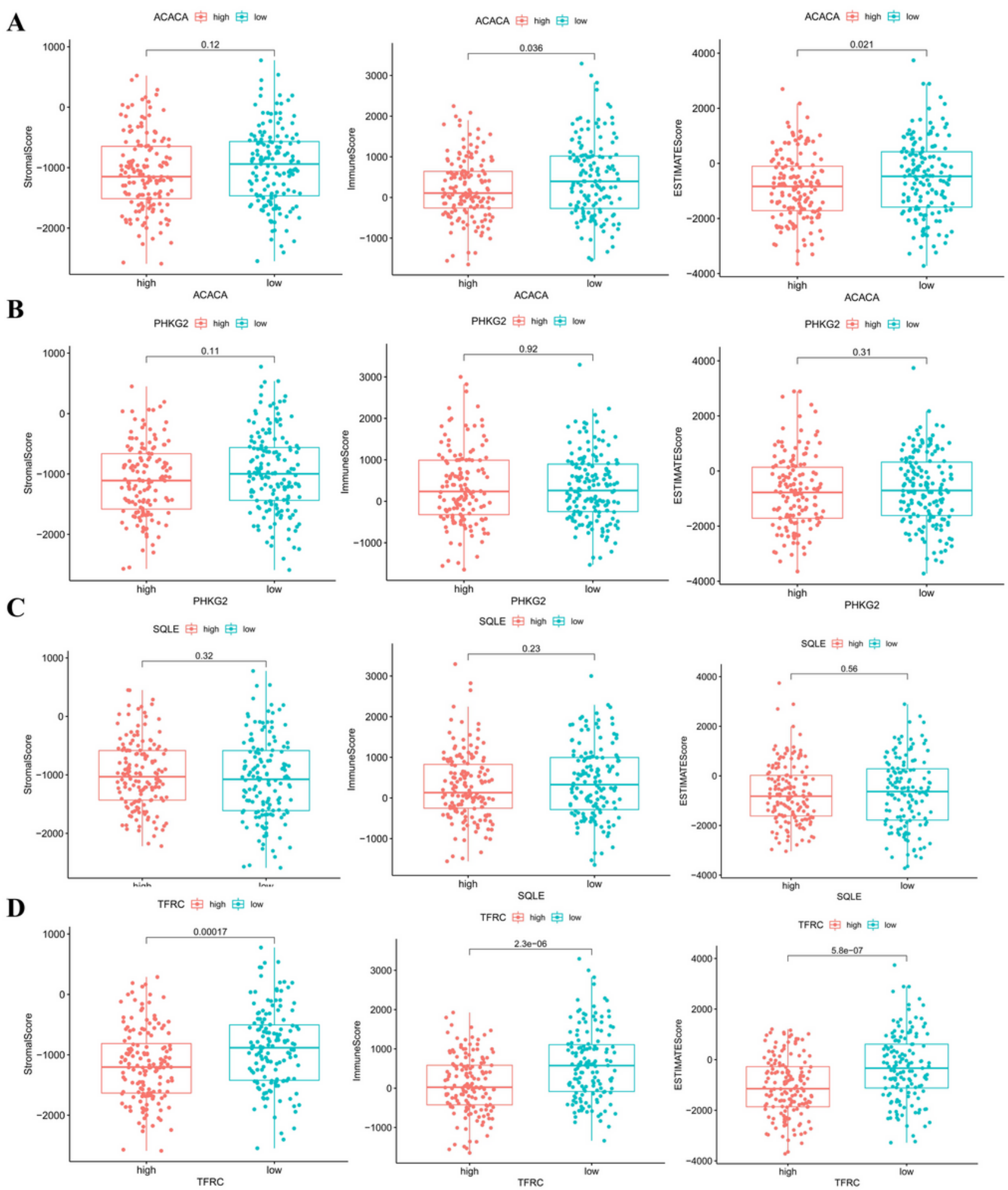

\section{Figure 5}

The correlation between tumor microenvironment score and 4 key prognostic-related DEFGs in CESC. A. The correlation between stromal score and the expression of ACACA. B. The correlation between stromal score and the expression of PHKG2. C. The correlation between stromal score and the expression of SQLE. D. The correlation between stromal score and the expression of TFRC. 

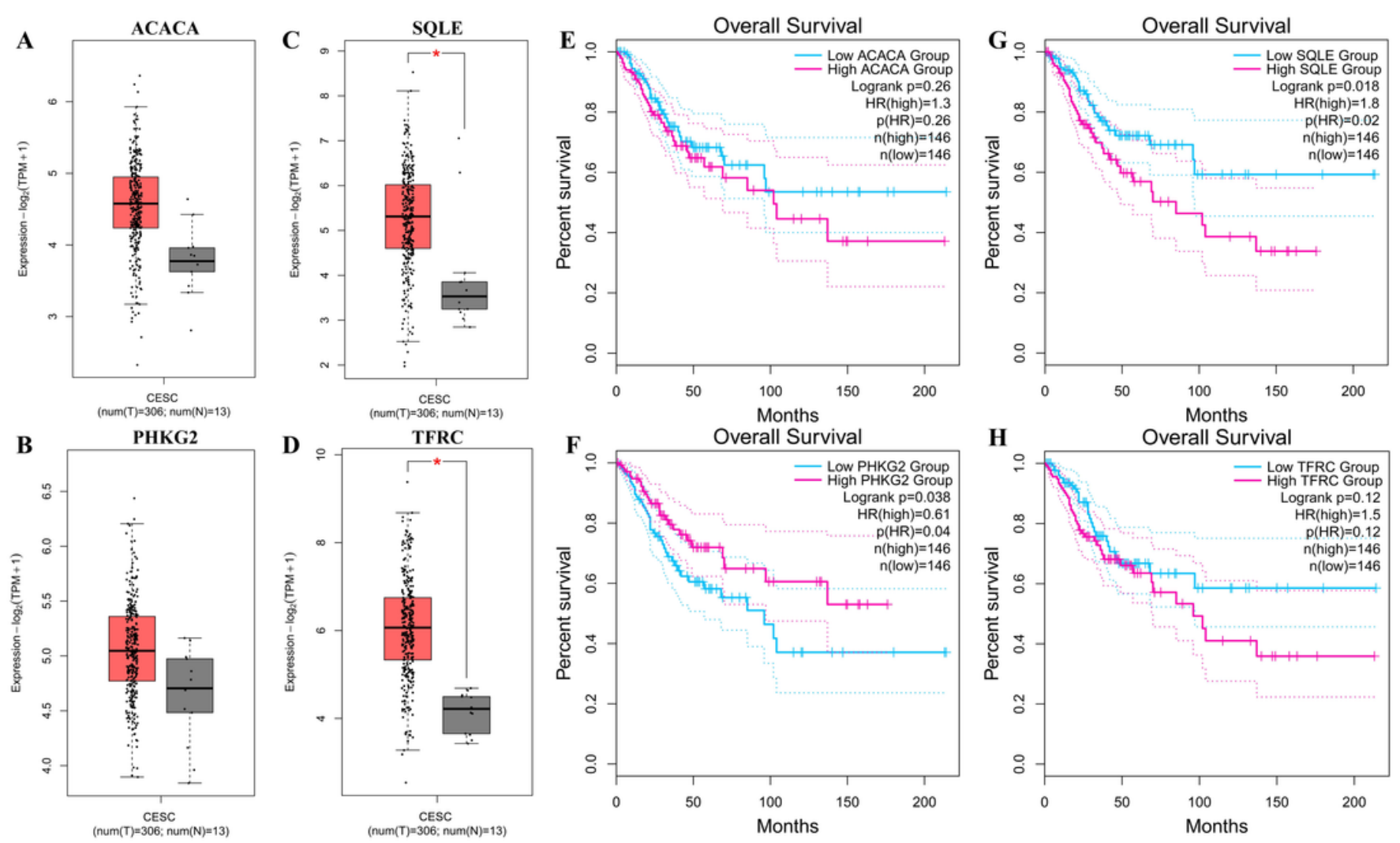

\section{Figure 6}

The verification of 4 key prognostic-related DEFGs in GEPIA database. A. The verification of ACACA expression. B. The verification of PHKG2 expression. C. The verification of SQLE expression. D. The verification of TFRC expression. $\mathbf{E}$. The verification of survival curve in ACACA. F. The verification of survival curve in PHKG2. G. The verification of survival curve in SQLE. $H$. The verification of survival curve in TFRC. 


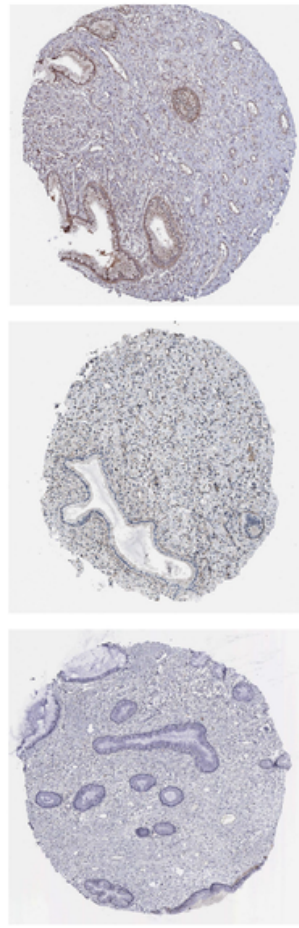

ACACA

Cervix normal tissue

Age: 26

Gender: Female

Patient id: 4490

Staining: Low

Intensity:Weak

Quantity: $>75 \%$

\section{SQLE}

Cervix normal tissue

Age: 26

Gender: Female

Patient id: 4490

Staining: Low

Intensity:Weak

Quantity: $>75 \%$

TFRC

Cervix normal tissue

Age: 27

Gender: Female

Patient id: 2004

Staining: Medium

Intensity: Moderate

Quantity: $>75 \%$
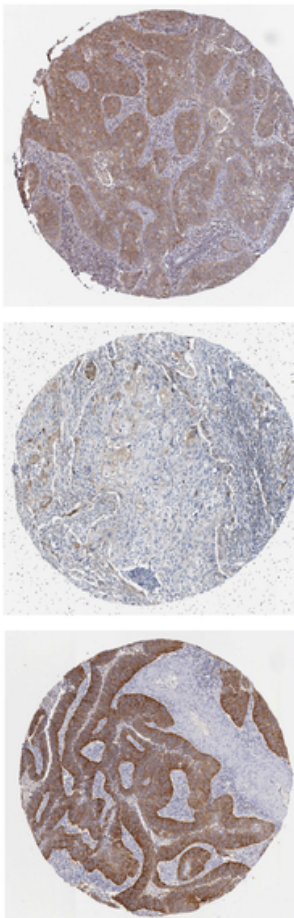

ACACA

Cervix SCC

Age: 31

Gender: Female

Patient id: 5104

Staining: Medium

Intensity: Moderate

Quantity: $>75 \%$

\section{SQLE}

Cervix SCC

Age: 70

Gender: Female

Patient id: 3382

Staining: Low

Intensity: Moderate

Quantity: $<25 \%$

\section{TFRC}

Cervix SCC

Age: 39

Gender: Female

Patient id: 1716

Staining: High

Intensity: Strong

Quantity: $>75 \%$

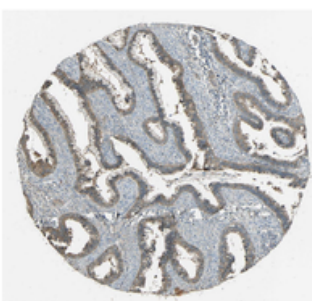

ACACA

Cervix adenocarcinoma Age: 44

Gender: Female

Patient id: 2480

Staining: Medium

Intensity: Moderate

Quantity: $>75 \%$

SQLE

Cervix adenocarcinoma

Age: 44

Gender: Female

Patient id: 2480

Staining: High

Intensity: Strong

Quantity: 75\%-25\%

\section{TFRC}

Cervix adenocarcinoma

Age: 44

Gender: Female

Patient id: 2480

Staining: High

Intensity: Strong

Quantity: $>75 \%$

Figure 7

The verification of ACACA, SQLE and TFRC in HPA database. 